\title{
Multi-epoch and multi-frequency VLBI observation of X-ray to gamma-ray flare in TeV blazar Mrk 421
}

\author{
Kotaro NIINUMA* \\ Graduate School of Science and Engineering, Yamaguchi University, Yoshida 1677-1, \\ Yamaguchi, Yamaguchi 753-8512, Japan \\ E-mail: niinumadyamaguchi-u.ac.jp
}

\section{Motoki KINO}

Korea Astronomy and Space Science Institute (KASI), 776, Daedeokdae-ro, Yuseong-gu, Daejeon, Republic of Korea 305-348

\section{Akihiro DOI}

The Institute of Space and Astronautical Science, Japan Aerospace Exploration Agency, 3-1-1 Yoshinodai, Chuou-ku, Sagamihara, Kanagawa 229-8510, Japan

\begin{abstract}
In early 2013, large X-ray and GeV gamma-ray flares occurred in TeV blazar Mrk 421. To investigate the spectral behavior in the radio core region of Mrk 421 after the large high-energy flare phenomenon, we carried out quasi-simultaneous multi-frequency $(2-43 \mathrm{GHz}$ ) dense monitor of Mrk 421 by using the very long baseline array (VLBA) immediately after the flare. During these VLBA observations, we detected not only flux variation but also clear spectral change. Especially VLBI spectra in 2013 June 16, which corresponds to the 1st X-ray flare seen in the period of our follow-up observation shows relatively flat spectrum compared with the one in 2013 May 22. In the high-energy active state of this source, we have found that there are two periods when the synchrotron self-absorption (SSA) turnover is seen at $\sim 8 \mathrm{GHz}$ and at less than $\sim 2 \mathrm{GHz}$.
\end{abstract}

12th European VLBI Network Symposium and Users Meeting

7-10 October 2014

Cagliari, Italy

${ }^{*}$ Speaker. 


\section{High energy active state in 2013 season}

A huge GeV gamma-ray flare was caught by Fermi-LAT in beginning of March 2013 [四]. After this exceptional enhancement in $\mathrm{GeV}$ band, multiple large flares were detected not only at X-ray (by using several astronomical X-ray instruments; Swift-BAT, $S$ wift-XRT, and MAXI-GSC) and gamma-ray (see FigureW).

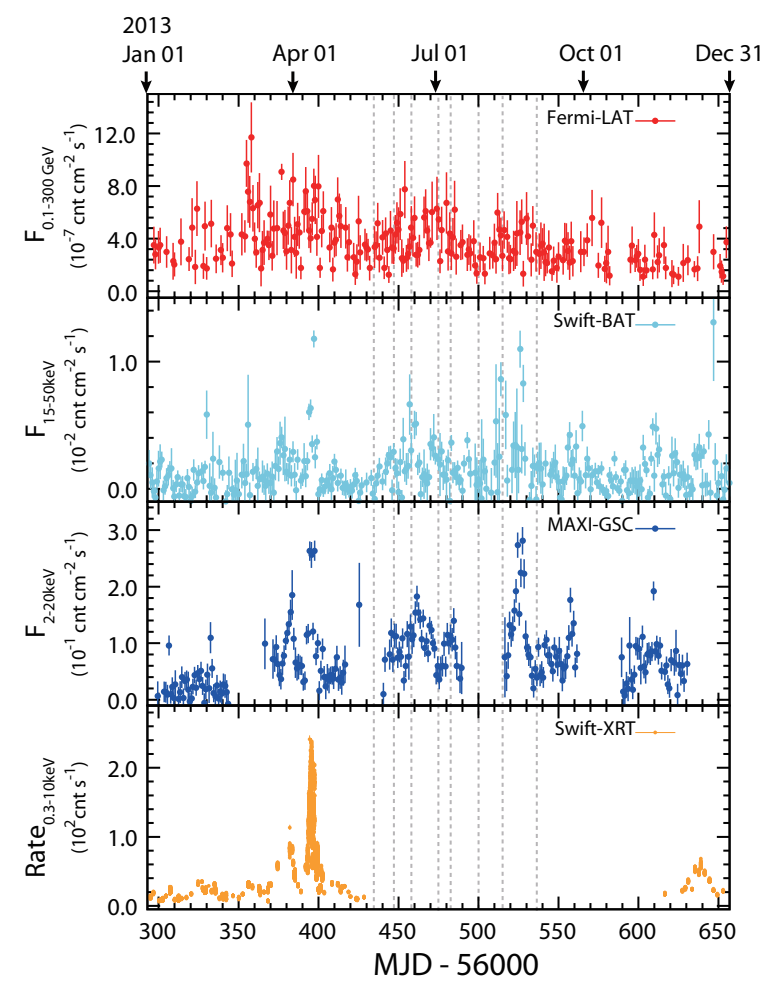

Figure 1: X-ray to GeV gamma-ray light curve of Mrk 421 in 2013. In beginning of March, a huge GeV gamma-ray flare was occurred, and then multiple flares were detected in X-ray band. Grey dotted lines represent the epochs when we conducted VLBA observations. Each light curve is derived from [0, 目, 团, 可]

\section{VLBA Observation}

We started follow-up observations from 2013 May 22 using the Very Long Baseline Array (VLBA) at 5-frequencies (2-43GHz) quasi-simultaneously. Observation summary are represented in Table $\mathbb{U}$. Each "Date" corresponds to grey dotted line shown in Figure $\mathbb{W}$. To focus on the quasi-simultaneous measurement of the flux density in the radio core region at multi-frequency, our observation time for each epoch is only 4.5 hours. Therefore $u v$-coverage of our observations is not suitable for detection of well-known extended faint jet structure of this source (see Figure [). Instead of detecting detailed structure, we obtained densely monitored data-set using VLBI. "High time-resolution" is key factor to discuss the spectral variation possibly associated with high energy flare phenomena.

\section{Results - Spectral variation}

Blazar spectral evolution associated with high-energy flare phenomena is densely studied 
Table 1: Observation Summary

\begin{tabular}{ccc}
\hline \hline Date & $\begin{array}{c}\text { Frequency } \\
\mathrm{GHz}\end{array}$ & Antennas $^{a}$ \\
\hline 2013 May 22 & $2.3,8.5,15.2,23.8,43.2$ & No SC \\
2013 Jun 06 & $2.3,8.5,15.2,23.8,43.2$ & No BR, FD, HN \\
2013 Jun 16 & $2.3,8.5,15.2,23.8,43.2$ & No FD \\
2013 Jul 03 & $2.3,8.5,15.2,23.8,43.2$ & No FD \\
2013 Jul 14 & $2.3,8.5,15.2,23.8,43.2$ & No FD, LA \\
2013 Jul 27 & $2.3,8.5,15.2,23.8,43.2$ & No FD \\
2013 Aug 11 & $2.3,8.5,15.2,23.8,43.2$ & No FD \\
2013 Sep 02 & $2.3,8.5,15.2,23.8,43.2$ & All \\
\hline
\end{tabular}

NOTES. ${ }^{a}$ BR: Brewster, Washington; FD: Fort Davis, Texas; HN: Hancock, New Hampshire; LA: Los Alamos, New Mexico; SC: Saint Croix, US Virgin Islands.

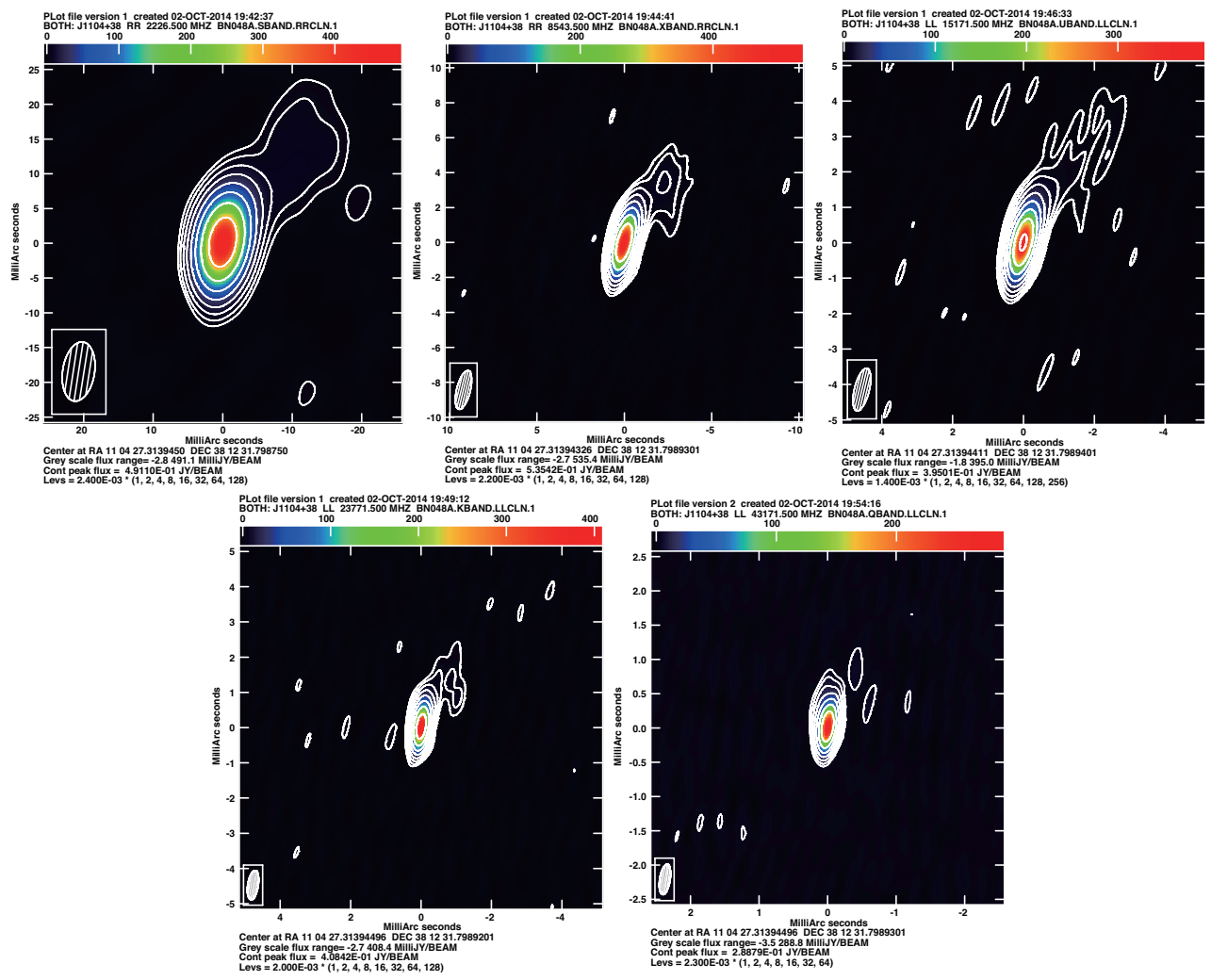

Figure 2: Multi-frequency VLBA images of Mrk 421 in 2013 May 22

based on single dish observations [ [ 6,0$]$. However, to identify the high energy flare location, VLBI measurement of spectral variation is crucial. For Mrk 421, this is the first quasi-simultaneous multifrequency VLBI observations to unveil the spectral evolution of sub-parsec to parsec scale region after high-energy flaring phenomenon. In our observations, we succeeded to detect not only flux variation and also spectral evolution (shift of tern-over frequency) between 2013 May and Sep in active state of Mrk 421 (see Figure [1]). VLBI spectra at the range between $2.3-43 \mathrm{GHz}$ in the 3rd 
epoch (2013 Jun 16) of our VLBA follow-up observations (the 3rd grey dashed line indicated in Figure $\mathbb{W}$ ), which corresponds to the first large X-ray flare seen during our observations, show relatively flat compared with the 1st epoch, and its ternover frequency is expected to shift to less than $2.3 \mathrm{GHz}$. Although the radio core region in this source shows optically thin spectra against synchrotron self-absorption at higher frequency than $\sim 10 \mathrm{GHz}$ on average based on previous VLBI studies [8, $[$, our result suggests that optically thick component also possibly appeared within the central sub-parsec region of Mrk 421 associated with the large high-energy flare.
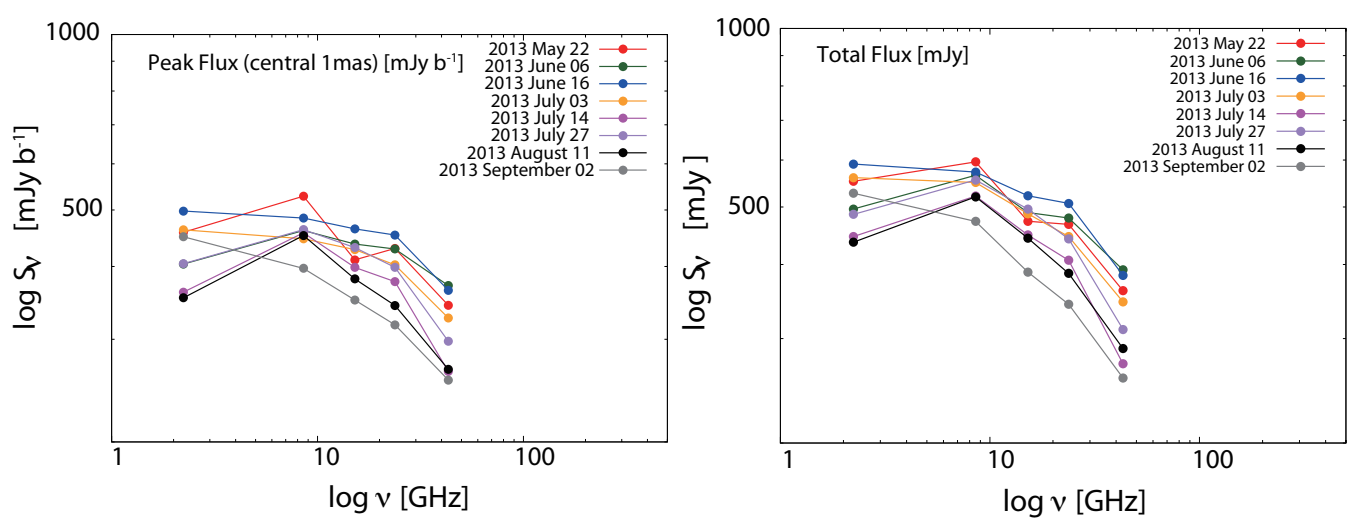

Figure 3: Spectral variation of Mrk 421 during our observations. To compare the flux density at each frequency, we restored the synthesized beam to a size of 1 mas (left panel). Here we compared the peak flux of central 1 mas region and the total flux (right panel) without error bars. These flux values are derived from preliminary analysis.

\section{Acknowledgement}

Swift-BAT transient monitor results provided by the $S$ wift-BAT team. This research has made use of the MAXI data provided by RIKEN, JAXA and the MAXI team.

\section{References}

[1] Fermi LAT weekly report N. 248 (http://fermisky.blogspot.jp/2013/03/fermi-lat-weekly-report-n-248.html)

[2] Fermi Science Support center (http://fermi.gsfc.nasa.gov/ssc/)

[3] Swift-BAT Hard X-ray Transient Monitor (http://swift.gsfc.nasa.gov/results/transients/; Krimm, H. A. et al., 2013, ApJSS 209, 14)

[4] MAXI-GSC data (http://maxi.riken.jp/top/; Matsuoka, M. et al., 2009, PASJ 61, 999)

[5] Swift-XRT Monitoring of Fermi-LAT Sources of Interest (http://www.swift.psu.edu/monitoring/; M.C. Stroh \& A.D. Falcone 2013, ApJS, 207, 28)

[6] Valtaoja, E. et al., 1988, A\&A 203, 1-20

[7] León-Tavares, J., et al., 2012, ApJ 754, 23

[8] Lico, R., et al., 2012, A\&A 545, A117.

[9] Blasi, M. G., et al., 2013, A\&A 559, 75. 